\title{
Do the benefits of sodium-glucose cotransporter 2 inhibitors exceed the risks in patients with type 1 diabetes?
}

\author{
Hikaru Kamoshima ${ }^{1)}$, Hiroshi Nomoto ${ }^{2}$, Kumiko Yamashita ${ }^{3)}$, Yuka Takahashi ${ }^{2)}$, Kazuhisa Tsuchida ${ }^{2)}$, \\ Saki Kuwabara ${ }^{2)}$, Aika Miya ${ }^{2)}$, Kyu Yong $\mathrm{Cho}^{2)}$, Hiraku Kameda ${ }^{2)}$, Akinobu Nakamura ${ }^{2)}$, Tatsuya Atsumi ${ }^{2)}$, \\ Shinji Taneda ${ }^{4)}$, Yoshio Kurihara ${ }^{3)}$, Shin Aoki ${ }^{5)}$, Yuri Ono ${ }^{1)}$ and Hideaki Miyoshi' ${ }^{2), 6}$ \\ 1) Yuri Ono Clinic, Diabetes, Internal Medicine, Sapporo 060-0001, Japan \\ 2) Department of Rheumatology, Endocrinology and Nephrology, Faculty of Medicine and Graduate School of Medicine, Hokkaido \\ University, Sapporo 060-8638, Japan \\ 3) Kurihara Clinic, Sapporo 004-0053, Japan \\ 4) Diabetes Center, Manda Memorial Hospital, Sapporo 060-0062, Japan \\ 5) Aoki Clinic, Sapporo 003-0023, Japan \\ 6) Division of Diabetes and Obesity, Faculty of Medicine and Graduate School of Medicine, Hokkaido University, Sapporo 060-8638, \\ Japan
}

\begin{abstract}
Sodium-glucose cotransporter 2 inhibitors (SGLT2is) are well-established means of improving glycemia and preventing cardio-renal events in patients with type 2 diabetes. However, their efficacy and safety have yet to be fully characterized in patients with type 1 diabetes (T1D). We studied patients with T1D who regularly attended one of five diabetes centers and treated with an SGLT2i (ipragliflozin or dapagliflozin) for $>52$ weeks, and the changes in HbA1c, body mass, insulin dose, and laboratory data were retrospectively evaluated and adverse events (AEs) recorded during December 2018 to April 2021. A total of 216 patients with T1D were enrolled during the period. Of these, 42 were excluded owing to short treatment periods and 15 discontinued their SGLT2i. The mean changes in glycated hemoglobin (HbA1c), body mass, and insulin dose were $-0.4 \%,-2.1 \mathrm{~kg}$, and $-9.0 \%$, respectively. The change in $\mathrm{HbAlc}$ was closely associated with the baseline HbA1c $(p<0.001)$, but not with the baseline body mass or renal function. The basal and bolus insulin doses decreased by $18.2 \%$ and $12.6 \%$, respectively, in participants with a baseline $\mathrm{HbA} 1 \mathrm{c}<8 \%$. The most frequent $\mathrm{AE}$ was genital infection $(2.8 \%)$, followed by diabetic ketoacidosis (DKA; $1.4 \%)$. None of the participants experienced severe hypoglycemic events. In conclusion, the administration of an SGLT2i in addition to intensive insulin treatment in patients with T1D improves glycemic control and body mass, without increasing the incidence of hypoglycemia or DKA.
\end{abstract}

Key words: Sodium-glucose cotransporter 2 inhibitor, Type 1 diabetes, Diabetic ketoacidosis

PATIENTS with type 1 diabetes (T1D) require lifelong insulin therapy to sustain life and to minimize the risk of hyperglycemic events, such as diabetic ketoacidosis (DKA). Intensive insulin therapy for good glycemic control reduces the risks of the development and progression of complications [1]. However, strict glycemic control is strongly associated with hypoglycemia and weight gain. In particular, the frequency of severe hypoglycemia is

Submitted Sep. 15, 2021; Accepted Oct. 28, 2021 as EJ21-0573

Released online in J-STAGE as advance publication Nov. 25, 2021 Correspondence to: Hideaki Miyoshi, MD, PhD, Division of Diabetes and Obesity, Faculty of Medicine and Graduate School of Medicine, Hokkaido University, N15 W7, Kita-ku, Sapporo 060-8638, Japan.

E-mail: hmiyoshi@med.hokudai.ac.jp high in patients with T1D. However, less than one-third of patients with T1D achieve their glycemic targets because of substantial glycemic variability caused by lower insulin secretion $[2,3]$. Therefore, new adjunctive therapies are required to improve glycemic control in patients with T1D without inducing hypoglycemia.

Sodium-glucose cotransporter 2 inhibitors (SGLT2is) improve glycemic control by reducing glycemic variability and decrease body mass by increasing urinary glucose excretion $[4,5]$. SGLT2is are recommended for those with type 2 diabetes (T2D) who are at high risk of cardiovascular disease, heart failure, or chronic kidney disease because consistent substantial benefits have been demonstrated in large-scale intervention studies [6]. Because the hypoglycemic effect of SGLT2is depends on 
urinary glucose excretion and, in turn, on blood glucose concentration and renal function, similar blood glucose and weight-reducing effects are also expected in patients with T1D $[7,8]$.

A meta-analysis of seven phase 2 or 3 clinical trials that compared the efficacy and safety of SGLT2is in patients with T1D showed that these drugs significantly reduced glycated hemoglobin (HbA1c) by $0.37 \%$ and body mass by $2.54 \mathrm{~kg}$ versus placebo, without increasing the incidence of hypoglycemia [9]. Two SGLT2is (ipragliflozin and dapagliflozin) are approved in Japan as adjunctive therapies to insulin for adults with T1D. Both SGLT2is significantly reduced HbA1c and body mass in a 24-week in a phase 3 trial in Japanese patients with T1D [10-12]. However, few studies have been performed to determine the durability of their effects in a routine clinical practice environment [13].

DKA is the most serious potential adverse event (AE) associated with the use of SGLT2 $i$ in patients with T1D and its incidence is more frequent in these patients than in those with T2D. In the present study, we aimed to evaluate the safety and efficacy of long-term SGLT2i use in Japanese patients with T1D in a real-world clinical setting and to determine whether the efficacy and safety of SGLT2is differ between subgroups of patients categorized according to their clinical characteristics.

\section{Materials and Methods}

\section{Study sample}

Patients with T1D who were undergoing multiple daily insulin therapy (MDI) or continuous subcutaneous insulin infusion (CSII) and who had been treated with an SGLT2i (ipragliflozin or dapagliflozin) for $>52$ weeks, up to April 2021, were eligible for inclusion. T1D was diagnosed by the attending physician and confirmed by depletion of insulin secretion by the measurement of plasma C-peptide concentration, clinical course, and/or presence of autoantibodies such as anti-GAD antibody. There was no restriction based on T1D subtype, but patients who were aged $<18$ years or pregnant were excluded. Patients were recruited at five medical centers and clinics specializing in diabetes located in Sapporo, Japan (Hokkaido University Hospital, Ono Yuri Clinic, Kurihara Clinic, Manda Memorial Hospital and Aoki Clinic).

\section{Study protocol}

We performed a multi-center, retrospective observational study, in which data were collected from the participants' medical records for the period between December 2018 and April 2021. The baseline was defined as the date that SGLT2i treatment was initiated.
Data were collected for each visit as follows: 4 weeks before the initiation of SGLT2i, at baseline, and 4, 12, and 52 weeks after starting the SGLT2i treatment. Ipragliflozin or dapagliflozin administration was initiated at $50 \mathrm{mg}$ or $5 \mathrm{mg}$, respectively, once daily after the first meal of the day. The primary endpoints of the study were the changes in HbAlc and body mass from baseline. The secondary endpoints were the changes in 1) daily insulin dose, 2) blood pressure, 3) serum creatinine concentration and estimated glomerular filtration rate, 4) urinary albumin/creatinine ratio, 5) liver enzyme activity, 6) hemoglobin and hematocrit, 7) factors associated with the changes in the primary endpoints, and 8) the incidences of any AEs.

Sub-analyses were also performed to determine the effects of SGLT2is on these parameters in participants with differing baseline HbA1c (A1, <8.0\%; A2, 8.0\%$8.9 \%$; $\mathrm{A} 3, \geq 9 \%$ ) and body mass index (BMI) (B1, $<22 \mathrm{~kg} / \mathrm{m}^{2}$; B2, 22-24.9 kg/m²; B3, $\geq 25 \mathrm{~kg} / \mathrm{m}^{2}$ ). To investigate the long-term safety of the use of SGLT2is in patients with T1D, AEs of interest and the discontinuation of the SGLT2i because of an AE were recorded during the study period. The AEs of interest were hypoglycemia, genital infection, and DKA.

An opt-out consent procedure was used. This study was registered with the University Hospital Medical Information Network (UMIN) Center (\#000044317), and the study protocol was approved by the Institutional Review Board of the Japan Clinicians Diabetes Association and Hokkaido University. The study was carried out in accordance with the principles of the Declaration of Helsinki, as revised in 2013.

\section{Statistical analysis}

Results are expressed as the mean \pm standard deviation for normally distributed data and as the median and $95 \%$ confidence interval $(95 \% \mathrm{CI})$ for non-normally distributed data. Categorical variables are expressed as numbers and percentages. Differences between two groups were evaluated using Student's $t$-test or the Wilcoxon signed-rank test for continuous variables. To compare multiple groups, we used one-way ANOVA, followed by Tukey's post-hoc test, or the Kruskal-Wallis test, followed by Dunn's multiple comparison test. Correlation coefficients and simple linear regression analyses were used to identify associations between variables. Multivariate linear regression analyses were used to identify factors that were independently associated with the outcomes. $p<0.05$ was considered to indicate statistical significance. All statistical analyses were performed using GraphPad Prism (GraphPad Software, Inc. San Diego, CA, USA) or EZR (Saitama Medical Center, Jichi Medical University, Saitama, Japan), which is a 
graphical user interface for $\mathrm{R}$ (The $\mathrm{R}$ Foundation for Statistical Computing, Vienna, Austria). More precisely, it is a modified version of $\mathrm{R}$ commander designed to add statistical functions that are frequently used in biostatistics.

\section{Results}

A total of 216 patients with T1D were treated with an SGLT2i during the study period. Of these, 42 who were continuing to take an SGLT2 $i$ at the time of data collection for the present study were excluded because of a short treatment period $(<52$ weeks). Fifteen patients were excluded because of discontinuation of their SGLT2 $\mathrm{i}$, owing to an $\mathrm{AE}(3.7 \%)$, ineffectiveness (1.4\%), pregnancy $(0.5 \%)$, or moving home $(1.4 \%)$, as shown in Supplementary Table 1. Of note, three participants experienced DKA. As a result, 159 participants who had been treated for $>52$ weeks were included in the efficacy analysis (Supplementary Fig. 1). The baseline clinical and metabolic characteristics of these 159 participants are shown in Supplementary Table 2. They comprised 106 women and 53 men, with a mean age of $50.7 \pm 15.1$ years and a mean duration of diabetes of $19.0 \pm 10.3$ years. All had been treated with MDI or CSII, and their median $(95 \%$ confidence interval, CI) total daily insulin dose prior to the start of SGLT2i therapy was 44.5 (40.5, 48.0) units/day.

Regular administration of an SGLT2i was initiated at baseline, and during the 52 weeks of SGLT2 $\mathrm{i}$ administration, the $\mathrm{HbAlc}(8.4 \% \pm 1.0 \%$ to $8.0 \% \pm 1.0 \%, p<$ $0.001)$ (Fig. 1A), body mass $(67.2 \pm 13.5 \mathrm{~kg}$ to 65.1 $\pm 13.3 \mathrm{~kg}, p<0.001)$ (Fig. 1B), and BMI $(25.5 \pm 4.2$ $\mathrm{kg} / \mathrm{m}^{2}$ to $\left.24.7 \pm 4.1 \mathrm{~kg} / \mathrm{m}^{2}, p<0.001\right)$ of the participants significantly decreased. The median doses of both bolus and basal insulin also significantly decreased (from 27.3 units to 24.0 units and from 16.0 units to 14.0 units, respectively, $p<0.001$ ), and as a result, the total insulin dose also decreased (from 44.5 units to 40.5 units, $p<$ 0.001) (Fig. 1C and Supplementary Table 2). The hemoglobin and hematocrit of the patients increased, whereas their circulating alanine aminotransferase (ALT) and gamma-glutamyl transferase $(\gamma \mathrm{GT})$ activities and uric acid concentration significantly decreased. Their estimated glomerular filtration rate (eGFR) decreased (from 85.0 $\pm 24.1 \mathrm{~mL} / \mathrm{min} / \mathrm{m}^{2}$ to $\left.78.8 \pm 21.4 \mathrm{~mL} / \mathrm{min} / \mathrm{m}^{2}, p<0.001\right)$, but there were no significant changes in their urinary albumin/creatinine ratio (UACR) or systolic or diastolic blood pressure (Supplementary Table 2).

To identify the factors associated with an improvement in HbA1c during treatment with an SGLT2i, we analyzed the relationships between the change in $\mathrm{HbA1c}$
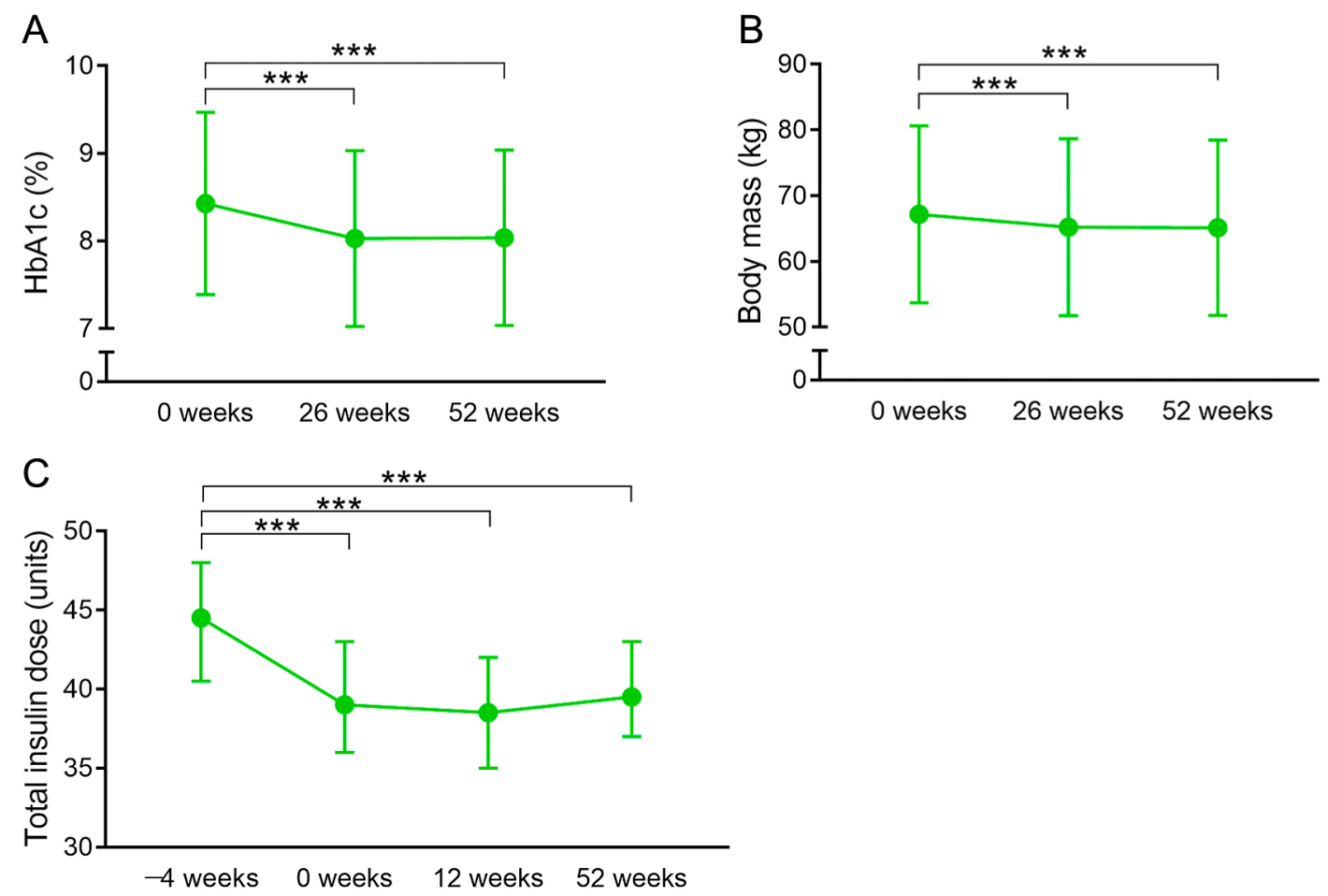

Fig. 1 Changes in HbA1c (\%) (A), body mass (kg) (B), and total insulin dose (units) (C) over the 52 weeks of the study Data are mean \pm standard deviation in (A) and (B), and median and $95 \%$ confidence in $(\mathrm{C})$. $* * * p<0.001$ vs. 0 weeks in (A) and (B), by Student's $t$-test; and $v s$. -4 weeks in (C), by the Wilcoxon signed-rank test. 
$(\triangle \mathrm{HbA} 1 \mathrm{c})$ and the baseline values of and changes in each parameter during the study. These analyses revealed a significant correlation between the reduction in $\mathrm{HbA} 1 \mathrm{c}$ and baseline $\mathrm{HbA} 1 \mathrm{c}(r=-0.46, p<0.001)$, but not baseline body mass ( $r=-0.11, p=0.19)$ (Fig. 2A and 2B). Multiple regression analysis showed that baseline $\mathrm{HbA} 1 \mathrm{c}$ and the percentage change in BMI $(\% \Delta \mathrm{BMI})$ were significantly associated with $\Delta \mathrm{HbA} 1 \mathrm{c}(p<0.001$ and $p=$ 0.001 , respectively) (Table 1 ).

The baseline clinical characteristics of the participants may have affected their changes in glycemic control, and therefore we next allocated the 159 participants to three groups based on their baseline HbA1c, as follows: A1 ( $n$ $=52, \mathrm{HbA} 1 \mathrm{c}<8 \%)$, A2 $(n=63$, HbAlc $8 \%-8.9 \%)$, and A3 ( $n=44, \mathrm{HbA} 1 \mathrm{c} \geq 9 \%)$. We found no significant differences in most of the baseline characteristics of the three sub-groups, with the exception of glycemic control, daily insulin dose, and insulin treatment regimen (Table 2). As expected, the effect of the SGLT2i on $\triangle \mathrm{HbA} 1 \mathrm{c}$ differed among the groups: it was minimal in A1, and significantly larger in the A2 and A3 groups (changes of $+0.1 \%,-0.5 \%$, and $-0.8 \%$, respectively) (Fig. 3). We also found that the percentage changes in the insulin doses differed among the sub-groups: the percentage changes in the total, basal, and bolus insulin doses during the study period were smaller in the $\mathrm{A} 3$ group than in the other two sub-groups (Table 3, Fig. 4A and B).

SGLT2is reduce the body mass of patients with T2D, and in general, patients with $\mathrm{T} 1 \mathrm{D}$ and obesity require a higher insulin dose. Therefore, we next determined the effect of SGLT2is in patients with T1D categorized according to their BMI: B1 $\left(n=32, \mathrm{BMI}<22 \mathrm{~kg} / \mathrm{m}^{2}\right)$, B2 $(n=43$, BMI 22-24.9 kg/m²), and B3 $(n=84$, BMI
A

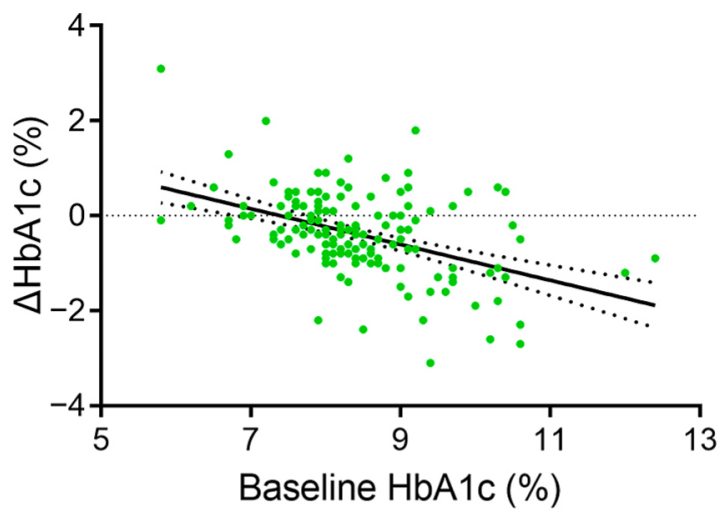

B

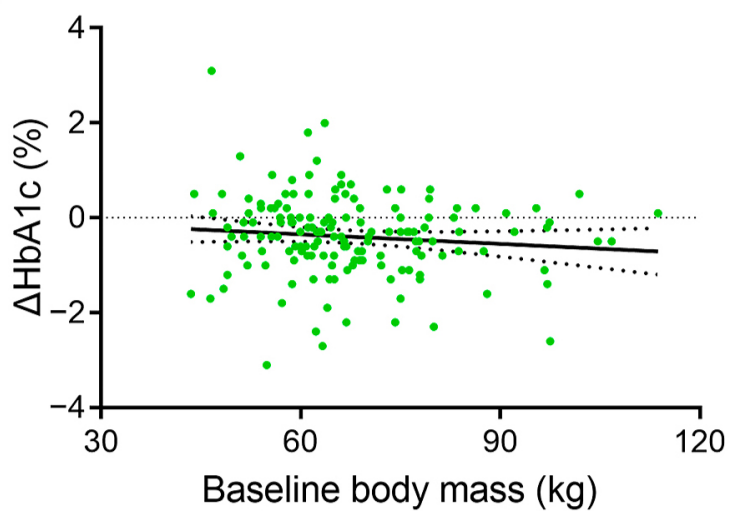

Fig. 2 Relationships of baseline HbAlc (\%) (A) and body mass (kg) (B) with the changes in HbAlc over the 52 weeks of the study

The reduction in $\mathrm{HbAlc}$ correlated with the baseline HbA1c $(r=-0.46 p<0.001)$, but not with baseline body mass.

Table 1 Relationships between changes in HbA1c during 1 year of treatment with an SGLT2i and clinical parameters, analyzed using multiple linear regression analysis

\begin{tabular}{lccc}
\hline Parameter & Regression coefficient & $95 \%$ confidence interval & $p$ value \\
\hline HbA1c $(\%)$ & -0.351 & $-0.480,-0.223$ & $<0.001$ \\
\hline$\% \Delta$ BMI $(\%)$ & -0.047 & $-0.075,-0.019$ & 0.001 \\
\hline BMI $\left(\mathrm{kg} / \mathrm{m}^{2}\right)$ & -0.029 & $-0.062,0.004$ & 0.08 \\
\hline Age (years) & -0.005 & $-0.016,-0.007$ & 0.40 \\
\hline Sex (female $=1)$ & 0.226 & $-0.063,0.516$ & 0.12 \\
\hline Duration of diabetes (years) & 0.008 & $-0.005,0.021$ & 0.22 \\
eGFR (mL/min/1.73 $\left.\mathrm{m}^{2}\right)$ & -0.006 & $-0.013,0.001$ & 0.12 \\
\hline Total insulin dose (units) & 0.003 & $-0.003,0.010$ & 0.23 \\
\hline$\% \Delta$ total insulin dose $(\%)$ & 0.003 & $-0.004,0.011$ & 0.33 \\
\hline
\end{tabular}

$R^{2}=0.3133$. The multiple linear regression analysis involved adjustment for age, sex, duration of diabetes, BMI, HbA1c, total insulin dose, eGFR, $\% \Delta \mathrm{BMI}$, and $\% \Delta$ total insulin dose. BMI, body mass index; eGFR, estimated glomerular filtration rate; $\% \triangle B M I$, percentage change in $B M I$ during the study; $\% \Delta$ total insulin dose, percentage change in daily total insulin dose during the study. 
Table 2 Baseline characteristics of the participants, categorized according to HbAlc

\begin{tabular}{|c|c|c|c|c|}
\hline & A1 group $(\mathrm{HbA} 1 \mathrm{c}<8 \%)$ & A2 group (HbA1c $8 \%-8.9 \%)$ & A3 group $(\mathrm{HbA} 1 \mathrm{c} \geq 9 \%)$ & $p$ value \\
\hline$n$ & 52 & 63 & 44 & \\
\hline Sex (male/female) & $17 / 35$ & $21 / 42$ & $15 / 29$ & 0.892 \\
\hline Age (years) & $51.1 \pm 16.6$ & $51.0 \pm 15.0$ & $49.8 \pm 13.6$ & 0.825 \\
\hline Duration of diabetes (years) & $19.8 \pm 12.2$ & $18.3 \pm 9.4$ & $19.0 \pm 9.6$ & 0.748 \\
\hline Body mass (kg) & $66.6 \pm 14.8$ & $65.6 \pm 10.5$ & $70.0 \pm 15.3$ & 0.239 \\
\hline Body mass index $\left(\mathrm{kg} / \mathrm{m}^{2}\right)$ & $25.2 \pm 4.5$ & $25.0 \pm 3.4$ & $26.5 \pm 4.8$ & 0.239 \\
\hline $\mathrm{HbA} 1 \mathrm{c}(\%)$ & $7.4 \pm 0.5$ & $8.3 \pm 0.3^{\dagger \dagger}$ & $9.7 \pm 0.8^{\dagger 十 \text { 柆 }}$ & $<0.001$ \\
\hline $\mathrm{CPG}(\mathrm{mg} / \mathrm{dL})$ & $187.7 \pm 83.8$ & $188.0 \pm 76.2$ & $221.6 \pm 86.6$ & 0.069 \\
\hline $\operatorname{eGFR}\left(\mathrm{mL} / \mathrm{min} / 1.73 \mathrm{~m}^{2}\right)$ & $81.0 \pm 27.0$ & $83.7 \pm 21.3$ & $90.4 \pm 25.0$ & 0.395 \\
\hline Creatinine $(\mathrm{mg} / \mathrm{dL})$ & $0.76 \pm 0.34$ & $0.69 \pm 0.17$ & $0.65 \pm 0.15$ & 0.443 \\
\hline Uric acid $(\mathrm{mg} / \mathrm{dL})$ & $4.4 \pm 1.5$ & $4.1 \pm 1.4$ & $4.4 \pm 1.2$ & 0.216 \\
\hline UACR (mg/gCre) & $8.7(4.0,17.0)$ & $7.4(4.4,19.5)$ & $9.9(4.1,24.6)$ & 0.851 \\
\hline AST (U/L) & $25.6 \pm 21.7$ & $20.4 \pm 6.1$ & $23.2 \pm 8.1$ & 0.111 \\
\hline ALT (U/L) & $20.8 \pm 13.4$ & $19.6 \pm 10.6$ & $23.7 \pm 12.1$ & 0.078 \\
\hline$\gamma \mathrm{GTP}(\mathrm{U} / \mathrm{L})$ & $25.0 \pm 23.2$ & $23.8 \pm 17.3$ & $40.4 \pm 51.6$ & 0.074 \\
\hline Hemoglobin (g/dL) & $13.8 \pm 1.4$ & $14.1 \pm 1.4$ & $14.2 \pm 1.3$ & 0.229 \\
\hline Hematocrit (\%) & $42.0 \pm 4.3$ & $45.1 \pm 4.5$ & $43.5 \pm 3.7$ & 0.140 \\
\hline Systolic BP (mmHg) & $127 \pm 13$ & $126 \pm 19$ & $124 \pm 16$ & 0.446 \\
\hline Diastolic BP (mmHg) & $75 \pm 9$ & $73 \pm 12$ & $71 \pm 8$ & 0.107 \\
\hline SGLT2i (Ipragliflozin/Dapagliflozin) & $26 / 26$ & $34 / 29$ & $22 / 22$ & 0.973 \\
\hline Retinopathy (NDR/SDR/PPDR/PDR) & $38 / 10 / 2 / 2$ & $47 / 14 / 1 / 1$ & $25 / 17 / 1 / 1$ & 0.347 \\
\hline Insulin regimen (CSII/MDI) & $8 / 29$ & $2 / 61$ & $1 / 43$ & 0.038 \\
\hline Total insulin dose (units) & $40.0(32.0,47.0)$ & $45.0(39.0,50.0)$ & $45.0(38.0,48.0)$ & 0.429 \\
\hline Basal insulin dose (units) & $12.0(11.0,14.0)$ & $18.0(14.0,20.0)$ & $18.0(16.0,22.0)^{\dagger}$ & 0.010 \\
\hline Bolus insulin dose (units) & $27.0(23.0,30.0)$ & $26.0(21.0,32.0)$ & $30.0(22.0,33.0)$ & 0.837 \\
\hline
\end{tabular}

Data are mean \pm standard deviation, median and $95 \%$ confidence interval, or numbers; and were analyzed using one-way ANOVA, followed by Tukey's post-hoc test, or the Kruskal-Wallis test, followed by Dunn's multiple comparison test. ${ }^{\dagger} p<0.05 v s$. A1, ${ }^{\dagger \dagger} p<0.01$ vs. A1, $p<0.01$ vs. A2. CPG, casual plasma glucose; eGFR, estimated glomerular filtration rate; UACR, urinary albumin/creatinine ratio; AST, aspartate aminotransferase; ALT, alanine aminotransferase; BP, blood pressure; SGLT2i, sodium-glucose cotransporter 2 inhibitor; NDR, no diabetic retinopathy; SDR, simple diabetic retinopathy; PPDR, pre-proliferative diabetic retinopathy; PDR, proliferative diabetic retinopathy; CSII, continuous subcutaneous insulin infusion; MDI, multiple daily insulin therapy.

$\left.\geq 25 \mathrm{~kg} / \mathrm{m}^{2}\right)$. The baseline body mass, BMI, and insulin dose of the B2 and B3 groups were higher than those of the B1group (all $p<0.001$ ), as shown in Supplementary Table 3. However, no significant differences were observed among the three groups with respect to other parameters, including sex, age, duration of diabetes, eGFR, UACR, and blood pressure. After SGLT2i treatment, $\mathrm{HbAlc}$ was significantly lower in all three of the groups and the reductions were similar in magnitude (Supplementary Fig. 2). Body mass and BMI also decreased in all three of the groups (Supplementary Fig. 3). The reduction in body mass appeared to be smaller in the B1 group. When we analyzed the relation- ships between the change in body mass and the baseline values, there was a trend toward a negative correlation, but this was not statistically significant $(p=0.09)$ (Supplementary Fig. 4). Participants with higher BMIs were administered larger doses of insulin at baseline, as expected (Supplementary Table 3), but the percentage changes $(\% \Delta)$ in insulin dose in the three groups were similar (Supplementary Table 4).

During the study period, the most frequent $\mathrm{AE}$ was genital infection (six events, $2.8 \%$ ), followed by DKA (three events, 1.4\%). Severe hypoglycemia was not identified and the incidence of hypoglycemia did not increase after treatment with SGLT2is. The details of the three 


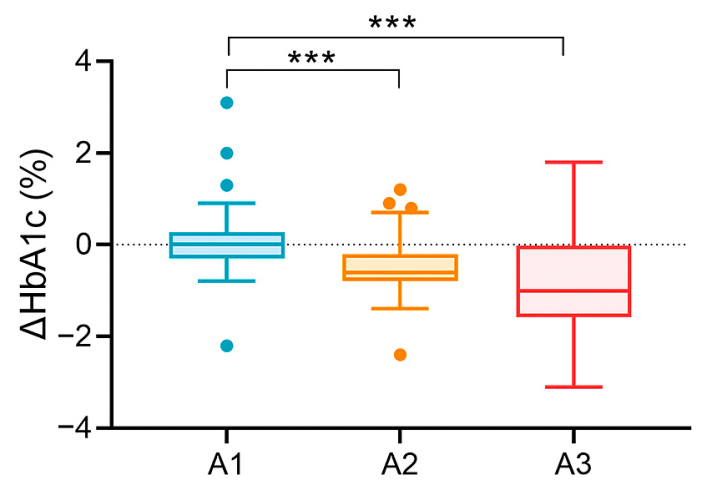

Fig. 3 Change in HbAlc (\%) over the 52 weeks of the study in participants categorized according to baseline $\mathrm{HbAlc}$ *** $p<0.001$, Kruskal-Wallis test followed by Dunn's multiple comparison test. A1, HbA1c $<8 \%$; A2, HbA1c $8 \%-8.9 \% ; \mathrm{A} 3, \mathrm{HbA} 1 \mathrm{c} \geq 9 \%$. cases of DKA recorded during the study are shown in Supplementary Table 5. These patients were treated appropriately while hospitalized and their SGLT2i was discontinued.

\section{Discussion}

The present study was the first to evaluate the safety and efficacy of long-term SGLT2i use in patients with T1D in a large-scale real-world clinical setting. The SGLT2is reduced HbA1c (by $0.4 \%$ ), body mass (by $2.1 \mathrm{~kg}$ ), and the requirement for insulin (by 9.0\%) over 52 weeks. Baseline HbA1c level was identified as a contributor to the magnitude of the reduction in $\mathrm{HbAlc}$, but body mass and renal function did not affect the change in HbA1c. The circulating liver enzyme activities and uric acid concentration were also significantly lower, as previously shown in patients with T2D $[14,15]$. There were

Table 3 Changes in insulin dose during the study in the subgroups A1, A2, and A3

\begin{tabular}{lcccc}
\hline & & 0 weeks & 12 weeks & 52 weeks \\
\hline $\begin{array}{l}\text { Total insulin dose } \\
\text { (\% change) }\end{array}$ & A1 & $-11.2(-14.3,-7.4)$ & $-14.3(-17.2,-11.1)$ & $-14.6(-20.7,-10.7)$ \\
& A2 & $-12.8(-16.0,-8.2)$ & $-13.7(-15.4,-10.9)$ & $-12.0(-15.4,-8.9)$ \\
& A3 & $-4.2(-11.8,0.0)^{\dagger,}$ & $-7.5(-13.6,0.0)$ & $-6.0(-12.5,0.0)^{\dagger \dagger}$ \\
$\begin{array}{l}\text { Basal insulin dose } \\
(\% \text { change })\end{array}$ & A1 & $-14.3(-16.7,-12.5)$ & $-16.7(-20.0,-14.3)$ & $-18.2(-26.8,-12.5)$ \\
& A2 & $-13.3(-16.7,-11.1)$ & $-18.2(-20.0,-16.7)$ & $-18.2(-23.3,-14.3)$ \\
Bolus insulin dose & A3 & $-7.3(-12.5,0.0)^{\dagger \dagger}$ & $-15.6(-18.8,0.0)$ & $-11.8(-15.8,0.0)^{\dagger, \dagger}$ \\
$(\%$ change $)$ & A1 & $-10.2(-15.0,-7.1)$ & $-12.7(-17.6,-8.3)$ & $-12.6(-18.4,-6.7)$ \\
& A2 & $-12.3(-15.8,-6.1)$ & $-10.7(-13.6,-5.1)$ & $-7.0(-13.6,0.0)$ \\
\hline
\end{tabular}

Data are median and 95\% confidence interval, and were analyzed using the Kruskal-Wallis test, followed by Dunn's multiple comparison test. ${ }^{\dagger} p<0.05 v s . \mathrm{A} 1,{ }^{\dagger \dagger} p<0.01 v s$. A1, ${ }^{\ddagger} p<0.05 v s$. A2. A1, HbA1c $<8 \%$; A2, HbA1c $8 \%-$ $8.9 \% ; \mathrm{A} 3, \mathrm{HbA} 1 \mathrm{c} \geq 9 \%$.

A

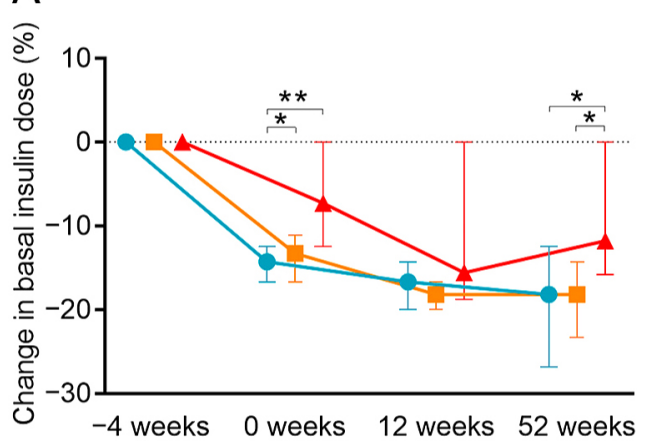

$\mathrm{B}$

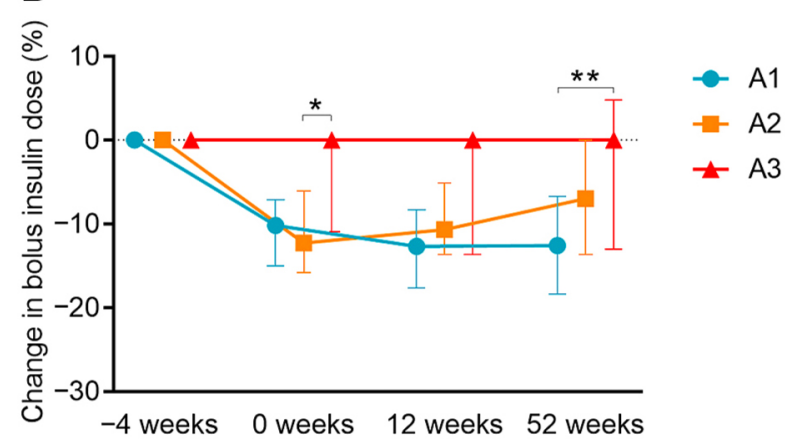

Fig. 4 Changes in basal (A) and bolus (B) insulin dose (percentage change from -4 weeks) over the 52 weeks of the study in participants categorized according to baseline $\mathrm{HbAlc}$

Data are median and 95\% confidence interval. A1 (blue circles and solid line), HbA1c <8\%; A2 (orange squares and solid line), HbA1c 8\%-8.9\%; A3 (red triangles and solid line), HbA1c $\geq 9 \%$. $* p<0.05, * * p<0.01$. 
markedly lower incidences of genital infection and DKA in these patients than has been previously reported $[9,12]$.

The improvement in HbA1c was comparable to those identified in phase 3 clinical trials conducted in Japanese patients with T1D (dapagliflozin: $-0.37 \%$ over 24 weeks; ipragliflozin: $-0.36 \%$ over 24 weeks and $-0.33 \%$ over 52 weeks) $[10,12,13]$ and a meta-analysis of studies conducted internationally [16] suggested that there are no differences in the overall HbAlc-lowering effect of SGLT2 is in patients of differing ethnicity with T1D. As has been shown in patients with T2D, as the HbA1c level at the baseline increased, the HbAlc-lowering effect of the SGLT2is increased. However, unlike in a previous study [7], the relationship between eGFR and the reduction in HbA1c was unclear (Table 1). This may be because most of the participants in the study had a normal eGFR and/or because of the smaller reductions in HbAlc achieved in the patients with T1D who were undergoing intensive insulin therapy, compared with that achieved in patients with T2D.

A single-center, retrospective observational study of 24-weeks SGLT2i use in 12 Japanese patients with T1D was reported very recently [17]. Although $\mathrm{HbAlc}$ reductions in the study was similar to those in phase 3 clinical trials, body mass reduction was relatively small $(-1.4 \mathrm{~kg})$. The reduction in body mass achieved in the present study was similar to those achieved in domestic and international phase 3 clinical trials of patients with T1D. Although a previous meta-analysis of clinical trials showed that SGLT2is reduced the body mass of patients with T1D (by a mean $-2.76 \mathrm{~kg}$ ) versus placebo, there was significant heterogeneity between the included studies [18]. The associations identified between weight loss while taking an SGLT2i and baseline body mass have been inconsistent in studies of patients with T2D [19]. In the present study of patients with T1D, we found that those with higher baseline body masses tended to lose more weight, but this trend was not significant $(p=0.09)$ (Supplementary Fig. 4).

A reduction in insulin dose should be considered when SGLT2is are co-administered to avoid hypoglycemia, but optimization of the dose is essential, because an excessive reduction increases the risk of developing DKA. In this study, the doses of basal and bolus insulin administered after SGLT2i treatment were determined by physicians according to the data from the Japanese Phase 3 clinical trials $[10,12,13]$ and the recommendations of the Japanese Diabetes Society (JDS) [20]. The mean reduction in insulin dose in the present study was similar to that in Japanese phase 3 clinical trials. The Japanese Diabetes Society (JDS) recommends that when adding an SGLT2 $i$ to the treatment regimen of patients with
T1D, especially if their glycemic control is good (HbA1c $<7.5 \%$ ), the total insulin dose should be reduced by $10 \%-20 \%$ [20]. Here, the reduction in bolus insulin dose in the A1 group (12.6\%) was larger than that in the A3 group (Table 3), and the reduction in the basal insulin dose in the A1 or A2 groups (18.2\%) was larger than that in the A3 group. The reductions in the basal insulin doses were larger than those in the bolus insulin doses in all three of the groups in the present study, similar to the findings of a previous study of patients with T2D [21]. No correlation was found between baseline BMI and the change in insulin dose in the present study (Supplementary Table 4).

In addition to their beneficial effects on HbAlc and weight control, SGLT2is improve daily and day-to-day glycemic variability [22, 23], and these would be the most significant benefits of using adjunctive therapy with an SGLT2i in patients with T1D. Indeed, most patients can feel their reduction of glycemic variability from the first day of administration, leading to an improvement in patient satisfaction and the costeffectiveness of therapy for T1D [24, 25]. Despite the described benefits of SGLT2is, most countries, including the United States, have not approved the use of SGLT2is for the treatment of T1D because of the higher risk of DKA.

A meta-analysis of eight international phase 2 and 3 trials conducted in patients with T1D showed that SGLT2 is are associated with a 5.8-times higher risk of DKA than placebo [16]. The European Medicines Agency has approved the drug for use only in patients with a BMI $\geq 27 \mathrm{~kg} / \mathrm{m}^{2}$ under specialist care [26]; this is because a phase 3 trial of Western patients with T1D showed that the incidence of DKA is higher in those with a BMI $<27 \mathrm{~kg} / \mathrm{m}^{2}$ than in those with a BMI $\geq 27 \mathrm{~kg} / \mathrm{m}^{2}$ (4.0\% vs. $1.7 \%)$ [27]. In contrast, Horii et al. [28] reported that SGLT2is increased the risk of DKA by 1.66-fold (hazard ratio 1.66; 95\% CI, 1.33-2.06; $p<$ 0001 ) based on an analysis of a database of 11,475 Japanese patients with T1D, considerably lower than in Western patients with T1D. Similarly, the incidences of DKA were low in the two phase 3 trials of the use of SGLT2is conducted in Japanese patients with T1D [10, 12, 13].

The discrepancy in the incidences of DKA between Western and Japanese patients cannot be inferred from these studies. However, one possible explanation is that the guidelines for reducing insulin dose in patients with T1D that were proposed early on by the JDS may have been appropriate. Alternatively, this may be because Japanese patients with T1D generally visit the hospital more frequently (usually every month) for check-ups, which may imply that their diabetes is more closely 
monitored than that of patients in other countries. Finally, intermittently scanned continuous glucose monitoring systems (iCGM) are now widely used for patients with T1D in Japan, which permits better selfmanagement by the patients. The use of iCGM was shown to be associated with a significantly lower incidence of DKA $(-56.2 \%)$ in patients with T1D [29].

In the present study of patients with $\mathrm{T} 1 \mathrm{D}$ and a mean BMI of $25.5 \mathrm{~kg} / \mathrm{m}^{2}$, DKA occurred in only three (1.4\%) over 52 weeks, which represents a lower incidence than that previously reported for patients with T1D who were not taking an SGLT2i (7.1\%) [28]. The three patients did not respond appropriately during sick days and had BMIs above the mean (Supplementary Table 5). DKA was more likely to occur during the initiation (insulin adjustment) period and was not associated with baseline BMI in a previous study of Japanese patients with T1D [28]. Therefore, we believe that there is no need to limit the use of SGLT2i to patients with T1D and obesity or overweight, as is the case in Europe, because they demonstrate efficacy in normal-weight patients. Importantly, the incidence of DKA is much higher in patients with T1D than in those with T2D because the risk is related to residual insulin secretory capacity $[30,31]$. Careful supervision by specialist clinicians, daily guidance regarding the sick day rule [32], and appropriate carbohydrate intake are crucial to reduce the risk of DKA in patients with T1D.

The present study had several limitations. First, it was a single-arm, retrospective observational study. Therefore, the results should be validated in a prospective study using a control group of patients with T1D who were not taking an SGLT2i. Second, because the analysis was conducted over a relatively short period of time (52 weeks), potential organ-protective effects, such as an improvement in renal function, were not evaluated. eGFR decreases during the first 6 months to 1 year of T2D [8], and thus a long-term observational study of patients with T1D and abnormal renal function should also be performed to determine whether SGLT2is have renal protective effects.

In conclusion, the addition of an SGLT2 $i$ to intensive insulin treatment in patients with T1D can provide additional benefits, such as improvements in glycemic control and body mass, without increasing the incidence of hypoglycemia or DKA. We found that the change in HbA1c in the participants was closely associated with the baseline HbA1c level, but not with body mass or renal function. Furthermore, the occurrence of DKA may not be predisposed to by low BMI and its incidence may be much lower in Japanese patients than in Western patients with T1D. Appropriate patient selection, continuous patient education, and risk reduction strategies, such as appropriate insulin dose adjustment, may improve the safety of the use of SGLT2is in patients with T1D and contribute to their long-term glycemic control and satisfaction.

\section{Acknowledgements}

The authors would like to express special gratitude to all of the patients and staff who contributed to this study. (Yuri Ono Clinic, Kurihara Clinic, Manda Memorial Hospital, Aoki Clinic and the Hokkaido University Hospital).

We also thank Mark Cleasby, PhD from Edanz (https://jp.edanz.com/ac) for editing a draft of this manuscript.

\section{Disclosure of Ethical Statements}

Approval of the research protocol: The study was approved by the Institutional Review Board of Japan Clinicians Diabetes Association and Hokkaido University.

Informed Consent: An opt-out consent procedure was used.

Approval date of Registry and the Registration No. of the study/trial: This study was registered with UMIN (\#000044317).

Animal Studies: N/A.

\section{Disclosure}

This study received no specific funding. Nakamura A, Atsumi T, Taneda S, Kurihara Y, and Miyoshi H have received research funding and received honoraria for lectures from some organizations as follows. The other authors declare that there is no conflict of interest. Nakamura A. has obtained research support from Novo Nordisk Pharma, MSD, Daiichi Sankyo Co. Ltd., Novartis Pharma, Mitsubishi Tanabe Pharma Co., AstraZeneca, Nippon Boehringer Ingelheim, LifeScan Japan, and Taisho Pharmaceutical. Atsumi $\mathrm{T}$ has accepted research grants and/or honoraria for meetings from GlaxoSmithKline K.K., Gilead Sciences Inc., Chugai Pharmaceutical Co., Ltd., Mitsubishi Tanabe Pharma Co., Astellas Pharma Inc., Pfizer Inc., Eisai Co.Ltd., Daiichi Sankyo Co. Ltd., AbbVie Inc., UCB Japan Co. Ltd., Bristol-Myers Squibb Co., Takeda Pharmaceutical Co., Ltd., Eli Lilly Japan K.K., Otsuka Pharmaceutical Co., Ltd., and Alexion Inc. Taneda S. has received honoraria for lectures from, Eli Lilly Japan K.K.,Ono Pharmaceutical Co., Ltd, and Novo Nordisk Pharma. Kurihara Y has received honoraria for lectures from MSD K.K., Ono Pharmaceutical Co., Ltd., Sanofi, 
Taisho Pharmaceutical Co., Ltd., Eli Lilly Japan K.K. , Sumitomo Dainippon Pharma Co. Ltd., and Takeda Pharmaceutical Co., Ltd. Miyoshi $\mathrm{H}$ has received honoraria for lectures from Eli Lilly Japan K.K., Astellas Pharma Inc., Mitsubishi Tanabe Pharma Co., Sumitomo Dainippon Pharma Co., Ltd., MSD K.K., Novo Nordisk Pharma, Nippon Boehringer Ingelheim Co., Kowa Pharmaceutical Co., Ltd., Sanofi, and Ono Pharmaceutical Co., Ltd., and has received research funding from Daiichi Sankyo Co., Sumitomo Dainippon Pharma Co. Ltd., Abbott Japan Co., Mitsubishi Tanabe Pharma Co., Eli Lilly Japan K.K., Novo Nordisk Pharma, Kowa Pharmaceutical Co., Ltd., Ono Pharmaceutical Co., Ltd., Nippon Boehringer Ingelheim Co., LifeScan Japan K.K., and Taisho Pharmaceutical Co., Ltd.

\section{Authorship}

YO and HM designed the study. HK, HN, and HM contributed to the writing of the manuscript and the data analysis. HK, HN, KY, YT, KT, SK, ST, YK, SA, and $\mathrm{HM}$ contributed to patient enrolment and collected data. $\mathrm{AM}, \mathrm{KYC}, \mathrm{HK}, \mathrm{AN}$, and TA reviewed and edited the manuscript. HM is the guarantor of this work and, as such, had full access to all the data and takes responsibility for the integrity of the data and the accuracy of the data analysis.

Supplementary Table 1 Reasons for discontinuation of the SGLT2i during the study

\begin{tabular}{lc}
\hline Reason for discontinuation & Number $(n=15$ total $)$ \\
\hline Inefficacy & 3 \\
Moving home or interruption of hospital visit & 3 \\
Diabetic ketoacidosis & 3 \\
Thirst and/or pollakiuria & 2 \\
Pregnancy & 1 \\
Fatigue & 1 \\
Rash & 1 \\
Nausea & 1 \\
\hline
\end{tabular}


Supplementary Table 2 Comparison of the characteristics of the participants at baseline and after 52 weeks of SGLT2i treatment

\begin{tabular}{|c|c|c|c|}
\hline & Baseline & 52 weeks & $p$ value \\
\hline Sex (male/female) & $53 / 106$ & & \\
\hline Age (year) & $50.7 \pm 15.1$ & & \\
\hline Duration of diabetes (years) & $19.0 \pm 10.3$ & & \\
\hline SGLT2i (Ipragliflozin/Dapagliflozin) & $82 / 77$ & & \\
\hline Retinopathy (NDR/SDR/PPDR/PDR) & $111 / 40 / 4 / 4$ & & \\
\hline Insulin (MDI/CSII) & $148 / 11$ & & \\
\hline Subtype (fulminant/acute/slowly progressive) & 9/107/43 & & \\
\hline Plasma C-peptide (ng/mL) & $0.04(0.02,0.38)$ & & \\
\hline Body mass (kg) & $67.2 \pm 13.5$ & $65.1 \pm 13.3$ & $<0.001$ \\
\hline Body mass index $\left(\mathrm{kg} / \mathrm{m}^{2}\right)$ & $25.5 \pm 4.2$ & $24.7 \pm 4.1$ & $<0.001$ \\
\hline HbA1c (\%) & $8.4 \pm 1.0$ & $8.0 \pm 1.0$ & $<0.001$ \\
\hline eGFR $\left(\mathrm{mL} / \mathrm{min} / 1.73 \mathrm{~m}^{2}\right)$ & $85.0 \pm 24.1$ & $78.8 \pm 21.4$ & $<0.001$ \\
\hline Creatinine (mg/dL) & $0.70 \pm 0.24$ & $0.75 \pm 0.30$ & $<0.001$ \\
\hline Uric acid $(\mathrm{mg} / \mathrm{dL})$ & $4.3 \pm 1.4$ & $4.1 \pm 1.3$ & $<0.01$ \\
\hline UACR (mg/gCre) & $8.1(4.0,21.4)$ & $9.2(4.3,22.1)$ & 0.78 \\
\hline AST (U/L) & $22.9 \pm 13.8$ & $21.1 \pm 8.6$ & 0.06 \\
\hline ALT (U/L) & $21.1 \pm 12.0$ & $19.1 \pm 11.0$ & $<0.01$ \\
\hline$\gamma \mathrm{GTP}(\mathrm{U} / \mathrm{L})$ & $28.7 \pm 32.4$ & $24.5 \pm 28.7$ & $<0.05$ \\
\hline Hemoglobin (g/dL) & $14.0 \pm 1.4$ & $14.5 \pm 1.5$ & $<0.001$ \\
\hline Hematocrit (\%) & $42.9 \pm 4.1$ & $44.5 \pm 4.6$ & $<0.001$ \\
\hline Systolic BP (mmHg) & $126 \pm 17$ & $124 \pm 16$ & 0.09 \\
\hline Diastolic BP (mmHg) & $73 \pm 10$ & $72 \pm 10$ & 0.61 \\
\hline Total insulin dose (units) & $44.5(40.5,48.0)$ & $40.5(37.0,43.0)$ & $<0.001$ \\
\hline Basal insulin dose (units) & $16.0(14.0,19.0)$ & $14.0(12.0,14.0)$ & $<0.001$ \\
\hline Bolus insulin dose (units) & $27.3(24.0,30.0)$ & $24.0(22.0,27.0)$ & $<0.001$ \\
\hline
\end{tabular}

Data are mean \pm standard deviation, median and $95 \%$ confidence interval, or number. Data were analyzed using Student's $t$-test or the Wilcoxon signed-rank test. SGLT2i, sodium-glucose cotransporter 2 inhibitor; MDI, multiple daily insulin therapy; CSII, continuous subcutaneous insulin infusion; NDR, no diabetic retinopathy; SDR, simple diabetic retinopathy; PPDR, pre-proliferative diabetic retinopathy; PDR, proliferative diabetic retinopathy; eGFR, estimated glomerular filtration rate; UACR, urinary albumin/creatinine ratio; AST, aspartate aminotransferase; ALT, alanine aminotransferase; BP, blood pressure. 
Supplementary Table 3 Baseline characteristics of the participants, categorized according to BMI

\begin{tabular}{|c|c|c|c|c|}
\hline & $\begin{array}{c}\text { B1 group } \\
\left(\mathrm{BMI}<22 \mathrm{~kg} / \mathrm{m}^{2}\right)\end{array}$ & $\begin{array}{c}\text { B2 group } \\
\left(\text { BMI } 22-25 \mathrm{~kg} / \mathrm{m}^{2}\right)\end{array}$ & $\begin{array}{c}\text { B3 group } \\
\left(\mathrm{BMI} \geq 25 \mathrm{~kg} / \mathrm{m}^{2}\right)\end{array}$ & $p$ value \\
\hline$n$ & 32 & 43 & 84 & \\
\hline Sex (male/female) & $7 / 25$ & $15 / 28$ & $31 / 53$ & 0.231 \\
\hline Age (years) & $52.2 \pm 14.6$ & $51.1 \pm 16.7$ & $49.9 \pm 14.6$ & 0.729 \\
\hline Duration of diabetes (years) & $17.0 \pm 10.3$ & $19.0 \pm 11.5$ & $19.7 \pm 9.7$ & 0.396 \\
\hline Body mass (kg) & $54.0 \pm 6.1$ & $62.7 \pm 7.3^{\dagger \dagger}$ & $74.6 \pm 13.1^{\dagger \dagger,+4 t}$ & $<0.001$ \\
\hline Body mass index $\left(\mathrm{kg} / \mathrm{m}^{2}\right)$ & $20.6 \pm 1.1$ & $23.4 \pm 0.8^{\dagger \dagger \dagger}$ & $28.5 \pm 3.3^{\dagger \dagger,++\hbar}$ & $<0.001$ \\
\hline HbA1c (\%) & $8.3 \pm 1.0$ & $8.4 \pm 1.0$ & $8.5 \pm 1.1$ & 0.416 \\
\hline Plasma glucose (mg/dL) & $197.5 \pm 80.7$ & $192.7 \pm 83.8$ & $199.4 \pm 83.5$ & 0.814 \\
\hline eGFR $\left(\mathrm{mL} / \mathrm{min} / 1.73 \mathrm{~m}^{2}\right)$ & $91.0 \pm 50.7$ & $88.2 \pm 25.8$ & $84.1 \pm 23.4$ & 0.418 \\
\hline Creatinine $(\mathrm{mg} / \mathrm{dL})$ & $0.71 \pm 0.28$ & $0.69 \pm 0.30$ & $0.71 \pm 0.19$ & 0.415 \\
\hline Uric acid (mg/dL) & $4.1 \pm 1.6$ & $4.1 \pm 1.2$ & $4.5 \pm 1.3$ & 0.038 \\
\hline UACR (mg/gCre) & $7.9(1.5-12.8)$ & $8.0(4.1-14.4)$ & $8.6(4.3-26.9)$ & 0.206 \\
\hline $\operatorname{AST}(\mathrm{U} / \mathrm{L})$ & $22.3 \pm 7.1$ & $22.6 \pm 16.2$ & $23.2 \pm 14.4$ & 0.279 \\
\hline $\operatorname{ALT}(\mathrm{U} / \mathrm{L})$ & $19.3 \pm 9.8$ & $19.7 \pm 12.5$ & $22.5 \pm 12.4$ & 0.463 \\
\hline$\gamma \mathrm{GTP}(\mathrm{U} / \mathrm{L})$ & $37.0 \pm 55.7$ & $25.4 \pm 30.9$ & $27.5 \pm 20.2$ & 0.075 \\
\hline Hemoglobin (g/dL) & $13.5 \pm 1.2$ & $13.9 \pm 1.3$ & $14.3 \pm 1.4$ & 0.018 \\
\hline Hematocrit (\%) & $41.1 \pm 3.5$ & $42.8 \pm 3.9$ & $43.7 \pm 4.2$ & 0.020 \\
\hline Systolic BP (mmHg) & $122 \pm 18$ & $127 \pm 18$ & $127 \pm 15$ & 0.389 \\
\hline Diastolic BP (mmHg) & $72 \pm 8$ & $73 \pm 12$ & $73 \pm 10$ & 0.953 \\
\hline SGLT2i (Ipragliflozin/Dapagliflozin) & $13 / 19$ & $22 / 21$ & $46 / 38$ & 0.214 \\
\hline Retinopathy (NDR/SDR/PPDR/PDR) & $23 / 8 / 0 / 1$ & $35 / 7 / 0 / 1$ & $52 / 26 / 4 / 2$ & 0.224 \\
\hline Insulin (CSII/MDI) & $1 / 31$ & $3 / 40$ & $7 / 77$ & 0.411 \\
\hline Total insulin dose (units) & $29.0(24.0,40.0)$ & $39.5(33.5,45.0)$ & $52.5(48.0,58.0)^{\dagger \dagger,+4}$ & $<0.001$ \\
\hline Basal insulin dose (units) & $11.0(8.0,14.0)$ & $13.0(11.0,16.0)$ & $20.0(18.0,22.0)^{\dagger \dagger,+1+t}$ & $<0.001$ \\
\hline Bolus insulin dose (units) & $18.0(14.0,25.0)$ & $26.0(22.2,30.0)$ & $32.0(27.0,36.0)^{\dagger \dagger \dagger}$ & $<0.001$ \\
\hline
\end{tabular}

Data are mean \pm standard deviation, median and $95 \%$ confidence, or number; and were analyzed using one-way ANOVA, followed by Tukey's post-hoc test, or the Kruskal-Wallis test, followed by Dunn's multiple comparison test. ${ }^{\dagger}, p<0.01 v s$. B1; ${ }^{\dagger \dagger}, p<0.001 v s$. B1; $p<0.01$ vs. B2; 林, $p<0.001$ vs. B2. BMI, body mass index; eGFR, estimated glomerular filtration rate; UACR, urinary albumin/ creatinine ratio; AST, aspartate aminotransferase; ALT, alanine aminotransferase; BP, blood pressure; SGLT2i, sodium-glucose cotransporter 2 inhibitor; NDR, no diabetic retinopathy; SDR, simple diabetic retinopathy; PPDR, pre-proliferative diabetic retinopathy; PDR, proliferative diabetic retinopathy; MDI, multiple daily insulin therapy; CSII, continuous subcutaneous insulin infusion. 
Supplementary Table 4 Changes in insulin doses during the study in the sub-groups B1, B2, and B3

\begin{tabular}{lcccc}
\hline & & 0 weeks & 12 weeks & 52 weeks \\
\hline $\begin{array}{l}\text { Total insulin dose } \\
(\% \text { change })\end{array}$ & B1 & $-7.9(-16.3,-4.2)$ & $-11.6(-16.0,-2.5)$ & $-10.8(-20.7,3.2)$ \\
& B2 & $-11.5(-16.0,-2.5)$ & $-15.1(-19.6,-11.3)$ & $-12.7(-15.7,-10.0)$ \\
& B3 & $-10.9(-13.9,-6.9)$ & $-12.5(-14.0,-8.6)$ & $-11.3(-14.6,-6.9)$ \\
$\begin{array}{l}\text { Basal insulin dose } \\
(\% \text { change })\end{array}$ & B1 & $-13.4(-18.2,-10.0)$ & $-20.0(-25.0,-14.3)$ & $-18.1(-26.8,-6.9)$ \\
& B2 & $-11.6(-16.7,-9.1)$ & $-18.2(-23.1,-14.3)$ & $-18.2(-25.0,-12.5)$ \\
Bolus insulin dose & B3 & $-13.8(-15.0,-11.1)$ & $-16.3(-17.9,-12.5)$ & $-15.2(-20.0,-10.0)$ \\
$(\%$ change $)$ & B1 & $-5.7(-16.7,0.0)$ & $-6.2(-12.5,0.0)$ & $-6.1(-15.2,6.1)$ \\
& B2 & $-12.3(-15.4,-7.1)$ & $-13.4(-17.6,-8.3)$ & $-9.6(-14.8,-4.8)$ \\
\hline
\end{tabular}

Data are median and 95\% confidence interval and were analyzed using the Kruskal-Wallis test, followed by Dunn's multiple comparison test. No significant differences were identified. B1, BMI $<22 \mathrm{~kg} / \mathrm{m}^{2}$, B2, BMI $22-25 \mathrm{~kg} / \mathrm{m}^{2} ; \mathrm{B} 3$, $\mathrm{BMI} \geq 25 \mathrm{~kg} / \mathrm{m}^{2}$.

Supplementary Table 5 Details of the three cases of diabetic ketoacidosis

\begin{tabular}{|c|c|c|c|}
\hline & Case 1 & Case 2 & Case 3 \\
\hline Sex & Female & Female & Male \\
\hline Age (years) & 46 & 42 & 68 \\
\hline Duration of diabetes (years) & 5 & 21 & 35 \\
\hline Insulin treatment regimen & MDI & CSII & MDI \\
\hline $\mathrm{HbA} 1 \mathrm{c}(\%)$ & 8.8 & 8.6 & 7.6 \\
\hline Body mass index $\left(\mathrm{kg} / \mathrm{m}^{2}\right)$ & 25.8 & 29.2 & 42.6 \\
\hline SGLT2i & Dapagliflozin & Ipragliflozin & Ipragliflozin \\
\hline Duration of SGLT2i use (weeks) & 28 & 28 & 16 \\
\hline Trigger for DKA & Gastroenteritis & Gastroenteritis & Appetite loss \\
\hline $\begin{array}{l}\text { Change in insulin dose after SGLT2 } \mathrm{i} \\
\text { introduced }\end{array}$ & -2 units & Unchanged & +17 units \\
\hline $\mathrm{pH}$ during DKA & 7.164 & 6.997 & 7.196 \\
\hline Plasma glucose during DKA (mg/dL) & 326 & 340 & 399 \\
\hline Treatment for DKA & $\begin{array}{c}\text { Stop SGLT2i } \\
\text { Insulin infusion } \\
\text { Dextrose infusion }\end{array}$ & $\begin{array}{c}\text { Stop SGLT2i } \\
\text { Insulin infusion } \\
\text { Dextrose infusion }\end{array}$ & $\begin{array}{c}\text { Stop SGLT2i } \\
\text { Insulin infusion } \\
\text { Dextrose infusion } \\
\text { Sodium bicarbonate infusion }\end{array}$ \\
\hline Duration of hospitalization (days) & 5 & 7 & 10 \\
\hline
\end{tabular}

Each case of DKA was treated by stopping the SGLT2i, insulin infusion, and dextrose infusion. MDI, multiple daily insulin therapy; CSII, continuous subcutaneous insulin infusion; SGLT2i, sodium-glucose co-transporter 2 inhibitor; DKA, diabetic ketoacidosis. 


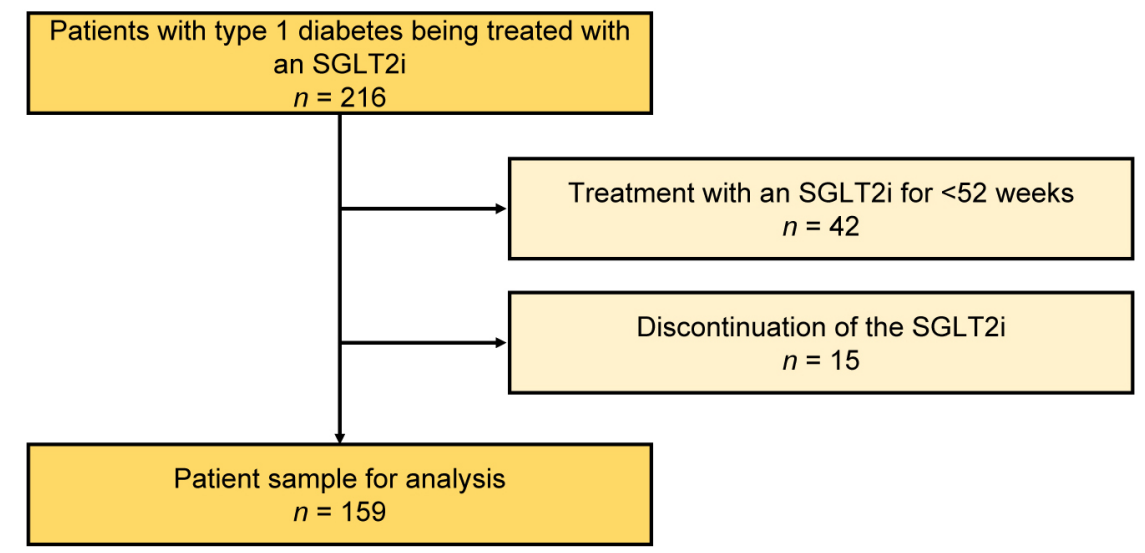

Supplementary Fig. 1 Flow diagram for the study

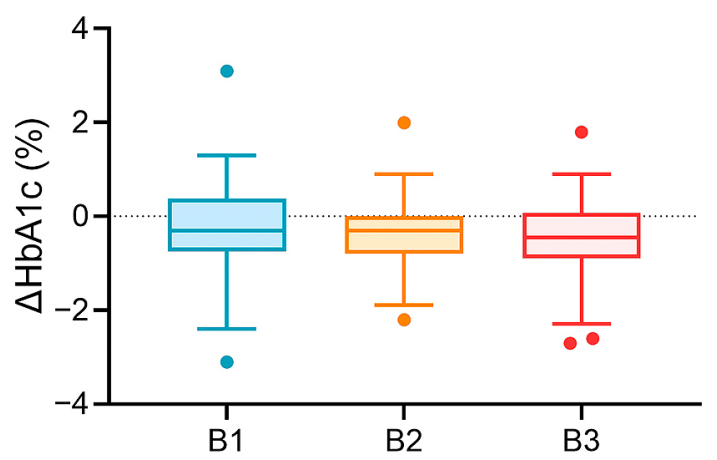

Supplementary Fig. 2 Changes in HbAlc (\%) over the 52 weeks of the study in participants categorized according to baseline body mass index (BMI)

Data were analyzed using the Kruskal-Wallis test. No significant differences were identified. B1, BMI $<22 \mathrm{~kg} / \mathrm{m}^{2}$, B2, BMI $22-25 \mathrm{~kg} / \mathrm{m}^{2} ; \mathrm{B} 3, \mathrm{BMI} \geq 25 \mathrm{~kg} / \mathrm{m}^{2}$.
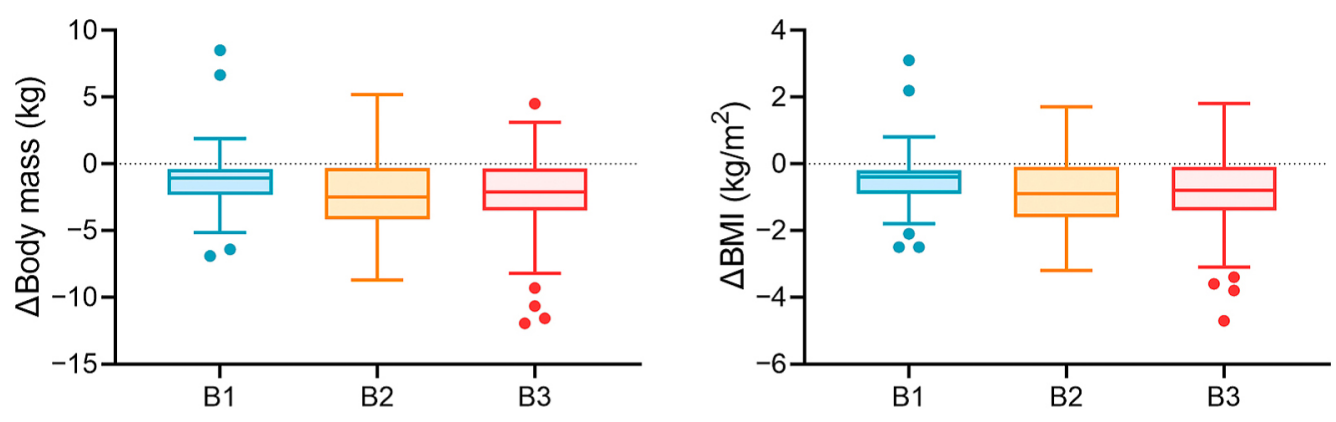

Supplementary Fig. 3 Changes in body mass and body mass index (BMI) over the 52 weeks of the study in participants categorized according to BMI category

Data were analyzed using the Kruskal-Wallis test. No significant differences were identified. B1, BMI $<22 \mathrm{~kg} / \mathrm{m}^{2}$, B2, BMI $22-25 \mathrm{~kg} / \mathrm{m}^{2} ; \mathrm{B} 3, \mathrm{BMI} \geq 25 \mathrm{~kg} / \mathrm{m}^{2}$. 


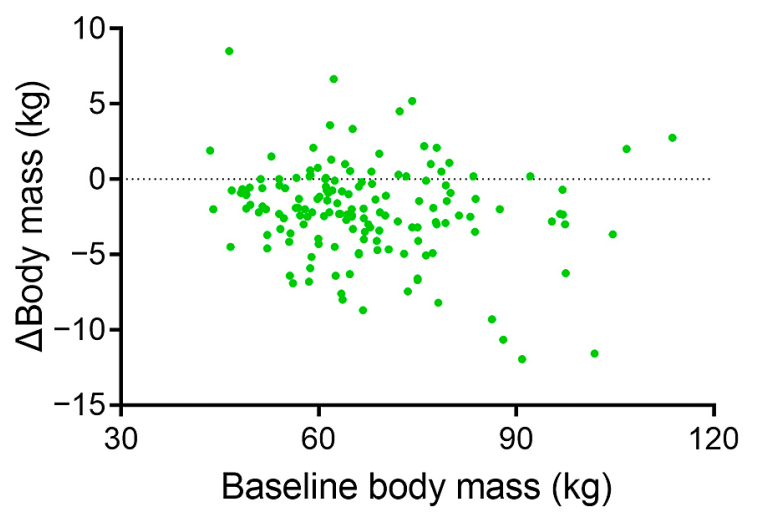

Supplementary Fig. 4 Relationship between baseline body mass $(\mathrm{kg})$ and the changes in body mass over the 52 weeks of the study A significant correlation was not identified.

\section{References}

1. Diabetes Control and Complications Trial Research Group, Nathan DM, Genuth S, Lachin J, Cleary P, et al. (1993) The effect of intensive treatment of diabetes on the development and progression of long-term complications in insulin-dependent diabetes mellitus. N Engl J Med 329: 977-986.

2. Gill G, Lucas S (1999) Brittle diabetes characterized by recurrent hypoglycaemia. Diabetes Metab 25: 308-311.

3. Foster NC, Beck RW, Miller KM, Clements MA, Rickels MR, et al. (2019) State of Type 1 Diabetes Management and Outcomes from the T1D Exchange in 2016-2018. Diabetes Technol Ther 21: 66-72.

4. Yamamoto C, Miyoshi H, Ono K, Sugawara H, Kameda $\mathrm{R}$, et al. (2016) Ipragliflozin effectively reduced visceral fat in Japanese patients with type 2 diabetes under adequate diet therapy. Endocr $J$ 63: 589-596.

5. Cho KY, Nomoto H, Nakamura A, Kawata S, Sugawara $\mathrm{H}$, et al. (2020) Favourable effect of the sodium-glucose co-transporter-2 inhibitor canagliflozin plus the dipeptidyl peptidase-4 inhibitor teneligliptin in combination on glycaemic fluctuation: an open-label, prospective, randomized, parallel-group comparison trial (the CALMER study). Diabetes Obes Metab 22: 458-462.

6. Buse JB, Wexler DJ, Tsapas A, Rossing P, Mingrone G, et al. (2020) 2019 update to: management of hyperglycemia in type 2 diabetes, 2018. A consensus report by the American Diabetes Association (ADA) and the European Association for the Study of Diabetes (EASD). Diabetes Care 43: 487-493.

7. Utsunomiya K, Shimmoto N, Senda M, Kurihara Y, Gunji $\mathrm{R}$, et al. (2017) Safety and effectiveness of tofogliflozin in elderly Japanese patients with type 2 diabetes mellitus : a post-marketing study (J-STEP/EL Study). J Diabetes Investig 8: 766-775.

8. Miyoshi H, Kameda H, Yamashita K, Nakamura A, Kurihara Y (2019) Protective effect of sodium-glucose cotransporter 2 inhibitors in patients with rapid renal func- tion decline, stage G3 or G4 chronic kidney disease and type 2 diabetes. J Diabetes Investig 10: 1510-1517.

9. Chen J, Fam F, Wang JY, Long Y, Gao CL, et al. (2017) The efficacy and safety of SGLT2 inhibitors for adjunctive treatment of type 1 diabetes: a systematic review and meta-analysis. Sci Rep 7: 44128.

10. Kaku K, Isaka H, Sakatani T, Toyoshima J (2019) Efficacy and safety of ipragliflozin add-on therapy to insulin in Japanese patients with type 1 diabetes mellitus: a randomized, double-blind, phase 3 trial. Diabetes Obes Metab 21: 2284-2293.

11. Araki E, Watada H, Uchigata $\mathrm{Y}$, Tomonaga O, Fujii H, et al. (2020) Efficacy and safety of dapagliflozin in Japanese patients with inadequately controlled type 1 diabetes (DEPICT-5): 52-week results from a randomized, openlabel, phase III clinical trial. Diabetes Obes Metab 22: 540-548.

12. Mathieu C, Dandona P, Gillard P, Senior P, Hasslacher C, et al. (2018) Efficacy and safety of dapagliflozin in patients with inadequately controlled type 1 diabetes (the DEPICT-2 Study): 24-week results from a randomized controlled trial. Diabetes Care 41: 1938-1946.

13. Kaku K, Isaka H, Sakatani T, Toyoshima J (2020) Longterm (52-week) efficacy and safety of ipragliflozin add-on therapy to insulin in Japanese patients with type 1 diabetes mellitus: an uncontrolled, open-label extension of a phase III study. J Diabetes Investig 11: 662-671.

14. Yamauchi Y, Nakamura A, Takahashi K, Takase T, Yamamoto C, et al. (2019) Factors with remission of fatty liver in patients with type 2 diabetes treated with ipragliflozin. Endocr J 66: 995-1000.

15. Takase T, Nakamura A, Miyoshi H, Yamamoto C, Atsumi $\mathrm{T}$ (2017) Amelioration of fatty liver index in patients with type 2 diabetes on ipragliflozin: an association with glucose-lowering effects. Endocr J 64: 363-367.

16. Taylor SI, Blau JE, Rother KI, Beitelshees AL (2019) SGLT2 inhibitors as adjunctive therapy for type 1 
diabetes: balancing benefits and risks. Lancet Diabetes Endocrinol 7: 949-958.

17. Shimoda M, Mashiko A, Katakura Y, Sanada J, Fushimi $\mathrm{Y}$, et al. (2021) Efficacy and safety of adding ipragliflozin to insulin in Japanese patients with type 1 diabetes mellitus: a retrospective study. Endocr $J$ doi: 10.1507/ endocrj.EJ21-0161. Online ahead of print.

18. El Masri D, Ghosh S, Jaber LA (2018) Safety and efficacy of sodium-glucose cotransporter 2 (SGLT2) inhibitors in type 1 diabetes : a systematic review and meta-analysis. Diabetes Res Clin Pract 137: 83-92.

19. Lee PC, Ganguly S, Goh SY (2018) Weight loss associated with sodium-glucose cotransporter-2 inhibition: a review of evidence and underlying mechanisms. Obes Rev 19: $1630-1641$.

20. Committee on the Proper Use of SGLT2 Inhibitors (2020) Recommendations on the proper use of SGLT2 inhibitors. J Diabetes Investig 11: 257-261.

21. Kanazawa K, Uchino H, Shigiyama F, Igarashi H, Ikehara $\mathrm{K}$, et al. (2019) Sustained fasting glucose oxidation and postprandial lipid oxidation associated with reduced insulin dose in type 2 diabetes with sodium-glucose cotransporter 2 inhibitor: a randomized, open-label, prospective study. J Diabetes Investig 10: 1022-1031.

22. Chiba K, Nomoto H, Nakamura A, Cho KY, Yamashita K, et al. (2021) Sodium-glucose cotransporter 2 inhibitors reduce day-to-day glucose variability in patients with type 1 diabetes. J Diabetes Investig 12: 176-183.

23. Mathieu C, Dandona P, Phillip M, Oron T, Lind M, et al. (2019) Glucose variables in type 1 diabetes studies with dapagliflozin: pooled analysis of continuous glucose monitoring data from DEPICT-1 and -2. Diabetes Care 42: 1081-1087.

24. Rodbard HW, Peters AL, Slee A, Cao A, Traina SB, et al. (2017) The effect of canagliflozin, a sodium glucose cotransporter 2 inhibitor, on glycemic end points assessed by continuous glucose monitoring and patient-reported outcomes among people with type 1 diabetes. Diabetes
Care 40: 171-180.

25. Bennett H, Tank A, Evans M, Bergenheim K, McEwan P, et al. (2020) Cost-effectiveness of dapagliflozin as an adjunct to insulin for the treatment of type 1 diabetes mellitus in the United Kingdom. Diabetes Obes Metab 22: 1047-1055.

26. (2021) Forxiga $5 \mathrm{mg}$ film-coated tablets: European Summary of Product Characteristics. European Medicine Agency. Surry, United Kingdom. https: //www.medicines. org.uk/emc/product/ 2865/smpc. accessed on Jul 31, 2021

27. Mathieu C, Dandona P, Birkenfeld AL, Hansen TK, Iqbal $\mathrm{N}$, et al. (2020) Benefit/risk profile of dapagliflozin $5 \mathrm{mg}$ in the DEPICT-1 and -2 trials in individuals with type 1 diabetes and body mass index $\geq 27 \mathrm{~kg} / \mathrm{m}^{2}$. Diabetes Obes Metab 22: 2151-2160.

28. Horii T, Oikawa Y, Atsuda K, Shimada A (2021) On-label use of sodium-glucose cotransporter 2 inhibitors might increase the risk of diabetic ketoacidosis in patients with type 1 diabetes. J Diabetes Investig 12: 1586-1593.

29. Roussel R, Riveline JP, Vicaut E, de Pouvourville G, Detournay B, et al. (2021) Important drop rate of acute diabetes complications in people with type 1 or type 2 diabetes after initiation of flash glucose monitoring in France: the RELIEF study. Diabetes Care 44: 1368-1376.

30. Misra S, Oliver NS (2015) Diabetic ketoacidosis in adults. BMJ 351: h5660.

31. Danne T, Garg S, Peters AL, Buse JB, Mathieu C, et al. (2019) International consensus on risk management of diabetic ketoacidosis in patients with type 1 diabetes treated with sodium-glucose cotransporter (SGLT) inhibitors. Diabetes Care 42: 1147-1154.

32. Goldenberg RM, Gilbert JD, Hramiak IM, Woo VC, Zinman B (2019) Sodium-glucose co-transporter inhibitors, their role in type 1 diabetes treatment and a risk mitigation strategy for preventing diabetic ketoacidosis: the STOP DKA protocol. Diabetes Obes Metab 21: 21922202. 